\title{
Ammonia Fiber Expansion Combined with White Rot Fungi to Treat Lignocellulose for Cultivation of Mushroom
}

Mingyang $\mathrm{Hu}^{\mathrm{a}}$, Lin Yuan ${ }^{\mathrm{a}}$, Ziyuan $\mathrm{Cai}^{\mathrm{a}}$, Jingjing Zhang ${ }^{\mathrm{a}}$, Dandan $\mathrm{Ji}^{\mathrm{a}, \mathrm{b}, \mathrm{c}, *}$, Lihua Zang ${ }^{\mathrm{a}, *}$

${ }^{a}$ College of Environmental Science and Engineering, Qilu University of Technology (Shandong Academy of Science), Jinan 250353, PR China

b Huatai Group, Guangrao 257335, PR China

c Jiangsu Key Laboratory of Anaerobic Biotechnology, Jiangnan University, Wuxi 214122, PR China

* Corresponding Author.

Tel: +86053189631680 ;

E-mail address: jdd@qlu.edu.cn; zlh@qlu.edu.cn. 
The following are three sets of data on the lignocellulose composition of the medium.

\section{Table S1}

Composition of cellulose, hemicellulose in the first group of experimental media.

\begin{tabular}{ccccccc}
\hline Number & $\begin{array}{c}\text { glucose } \\
\text { concentration } \mathrm{mg} / \mathrm{l}\end{array}$ & $\begin{array}{c}\text { xylose concentration } \\
\mathrm{mg} / \mathrm{l}\end{array}$ & $\begin{array}{c}\text { arabinose } \\
\mathrm{mg} / \mathrm{l}\end{array}$ & $\begin{array}{c}\text { Sample mass } \\
(\mathrm{mg})\end{array}$ & $\begin{array}{c}\text { Cellulose content } \\
(\%)\end{array}$ & $\begin{array}{c}\text { Hemicellulose content } \\
(\%)\end{array}$ \\
\hline PS & 1605.32380 & 686.52244 & 134.19103 & 301 & 44.68 & 23.53 \\
AFEXPS & 1511.25534 & 677.77269 & 122.53522 & 301 & 42.06 & 22.87 \\
SL & 1376.64513 & 616.2521 & 153.26154 & 302 & 38.19 & 22.23 \\
AFEXSL & 1432.35924 & 611.41728 & 143.52239 & 304 & 39.48 & 21.61 \\
PC & 1501.42266 & 612.12697 & 123.37510 & 300 & 41.93 & 21.19 \\
AFEXPC & 1496.63592 & 610.25042 & 125.61942 & 300 & 41.80 & 21.22 \\
\hline
\end{tabular}

\section{Table S2}

Composition of lignin in the first group of experimental media.

\begin{tabular}{cccccc}
\hline Number & Sample mass $(\mathrm{mg})$ & $105^{\circ} \mathrm{C}$ drying quality $(\mathrm{g})$ & $575^{\circ} \mathrm{C}$ drying quality $(\mathrm{g})$ & $\begin{array}{c}\text { Difference in quality before } \\
\text { and after drying }\end{array}$ & $\begin{array}{c}\text { Lignin content }(\%) \\
(\mathrm{g})\end{array}$ \\
\hline PS & 301 & 40.01989 & 39.94623 & 0.07366 & 24.47 \\
AFEXPS & 301 & 41.36798 & 41.29218 & 0.07580 & 25.18 \\
SL & 302 & 39.61836 & 39.53246 & 0.08590 & 28.44 \\
AFEXSL & 304 & 39.75696 & 39.67402 & 0.08294 & 27.28 \\
PC & 300 & 39.31876 & 39.22932 & 0.08944 & 29.81 \\
AFEXPC & 300 & 39.82984 & 39.74289 & 0.08695 & 28.98 \\
\hline
\end{tabular}




\section{Table S3}

Composition of cellulose, hemicellulose in the second group of experimental media.

\begin{tabular}{ccccccc}
\hline Number & $\begin{array}{c}\text { glucose } \\
\text { concentration } \mathrm{mg} / \mathrm{l}\end{array}$ & $\begin{array}{c}\text { xylose concentration } \\
\mathrm{mg} / \mathrm{l}\end{array}$ & $\begin{array}{c}\text { arabinose } \\
\mathrm{mg} / \mathrm{l}\end{array}$ & $\begin{array}{c}\text { Sample mass } \\
(\mathrm{mg})\end{array}$ & $\begin{array}{c}\text { Cellulose content } \\
(\%)\end{array}$ & $\begin{array}{c}\text { Hemicellulose content } \\
(\%)\end{array}$ \\
\hline PS & 1580.52683 & 653.52513 & 166.59164 & 301 & 43.99 & 23.80 \\
AFEXPS & 1537.15918 & 634.37270 & 112.03314 & 301 & 42.79 & 21.31 \\
SL & 1436.44901 & 604.514 & 144.11071 & 302 & 39.85 & 21.59 \\
AFEXSL & 1435.66634 & 590.81011 & 130.71346 & 304 & 39.57 & 20.60 \\
PC & 1539.62869 & 603.23244 & 110.32510 & 300 & 43.00 & 20.47 \\
AFEXPC & 1530.33231 & 602.44137 & 108.92647 & 300 & 42.74 & 20.40 \\
\hline
\end{tabular}

Table S4

Composition of lignin in the second group of experimental media.

\begin{tabular}{cccccc}
\hline Number & Sample mass $(\mathrm{mg})$ & $105^{\circ} \mathrm{C}$ drying quality $(\mathrm{g})$ & $575^{\circ} \mathrm{C}$ drying quality $(\mathrm{g})$ & $\begin{array}{c}\text { Difference in quality before } \\
\text { and after drying }\end{array}$ & Lignin content $(\mathrm{g})$ \\
\hline PS & 301 & 40.01513 & 39.94565 & 0.06948 & 23.08 \\
AFEXPS & 301 & 41.36991 & 41.29703 & 0.07288 & 24.21 \\
SL & 302 & 39.61656 & 39.53345 & 0.08311 & 27.52 \\
AFEXSL & 304 & 39.75595 & 39.67441 & 0.08154 & 26.82 \\
PC & 300 & 39.31671 & 39.22959 & 0.08712 & 29.04 \\
AFEXPC & 300 & 39.82913 & 39.74297 & 0.08616 & 28.72 \\
\hline
\end{tabular}




\section{Table S5}

Composition of cellulose, hemicellulose in the third group of experimental media.

\begin{tabular}{ccccccc}
\hline Number & $\begin{array}{c}\text { glucose } \\
\text { concentration } \mathrm{mg} / \mathrm{l}\end{array}$ & $\begin{array}{c}\text { xylose concentration } \\
\mathrm{mg} / \mathrm{l}\end{array}$ & $\begin{array}{c}\text { arabinose } \\
\mathrm{mg} / \mathrm{l}\end{array}$ & $\begin{array}{c}\text { Sample mass } \\
(\mathrm{mg})\end{array}$ & $\begin{array}{c}\text { Cellulose content } \\
(\%)\end{array}$ & $\begin{array}{c}\text { Hemicellulose content } \\
(\%)\end{array}$ \\
\hline PS & 1618.71994 & 638.12244 & 147.43104 & 301 & 45.06 & 22.69 \\
AFEXPS & 1569.44998 & 589.67356 & 104.42457 & 301 & 43.68 & 19.82 \\
SL & 1494.74899 & 613.2383 & 123.56071 & 302 & 41.47 & 21.08 \\
AFEXSL & 1465.75926 & 569.40998 & 118.72979 & 304 & 40.40 & 19.59 \\
PC & 1559.22782 & 601.61554 & 104.47622 & 300 & 43.54 & 20.21 \\
AFEXPC & 1569.32922 & 590.04335 & 110.02515 & 300 & 43.83 & 20.10 \\
\hline
\end{tabular}

Table S6

Composition of lignin in the third group of experimental media.

\begin{tabular}{cccccc}
\hline Number & Sample mass $(\mathrm{mg})$ & $105^{\circ} \mathrm{C}$ drying quality $(\mathrm{g})$ & $575^{\circ} \mathrm{C}$ drying quality $(\mathrm{g})$ & $\begin{array}{c}\text { Difference in quality before } \\
\text { and after drying }\end{array}$ & Lignin content $(\mathrm{g})$ \\
\hline PS & 301 & 40.00233 & 39.92556 & 0.07677 & 25.50 \\
AFEXPS & 301 & 41.36558 & 41.29469 & 0.07089 & 23.55 \\
SL & 302 & 39.61317 & 39.53899 & 0.07418 & 24.56 \\
AFEXSL & 304 & 39.75550 & 39.67487 & 0.08063 & 26.52 \\
PC & 300 & 39.31504 & 39.22966 & 0.08538 & 28.46 \\
AFEXPC & 300 & 39.82953 & 39.74477 & 0.08476 & 28.25 \\
\hline
\end{tabular}

